\section{THU0129 ASSESSMENT OF LONELINESS IN PATIENTS WITH INFLAMMATORY ARTHRITIS}

Hakan Emmungil ${ }^{1}$, Ufuk İlgen ${ }^{1}$, Sezin Turan ${ }^{1}$, Özge Kllıç2. ${ }^{1}$ Trakya University, Edirne, Turkey, ${ }^{2}$ Koç University Hospital, Psychiatry, İstanbul, Turkey

Background: Rheumatic disease and psychosocial status have bi-directional impacts on each other. Loneliness, as a component of psychosocial status, may be interrelated with affect and social support and may also be influenced by the disease itself in patients with inflammatory arthritis ${ }^{1}$. Objectives: The aim of this study is to document loneliness and associated factors in patients with inflammatory arthritis. Effects of demographic and disease-related factors, functional status, affect, and social support on loneliness, and loneliness on functional status will be evaluated.

Methods: Consecutive patients with rheumatoid arthritis(RA), ankylosing spondylitis(AS), and psoriatic arthritis(PsA), meeting ACR, ASAS, and CASPAR criteria, respectively, were included in the study. Demographic data and general clinical parameters(Table 1) were identified for each patient. Beck depression and Beck anxiety inventory, revised multidimentional scale of percieved social support, HAQ-DI, and UCLA loneliness scale (ULS-8), all validated in Turkish population, were used for the assessments. Nonparametric comparison (Wilcoxon-Mann-Whitney and Kruskal-Wallis) and correlation(Spearman) tests were used to evaluate associations of demographic data, clinical parameters, and depression, anxiety, social support, HAQ-DI scores with ULS-8 score. Multiple regression models were generated for significant associations.

Results: Demographic data, general clinical features, functional status, and ULS-8 scores of disease groups are summarized in Table 1. Among demographic and general clinical parameters, higher number of total drugs and lower education were associated with significantly higher ULS8 scores (data not shown). Although weak, there were significant correlations between ULS-8 and HAQ-DI, depression, anxiety, social support, and doctor global VAS scores (Table 2). Stronger correlations were observed between HAQ-DI and depression, anxiety, and patient global VAS scores (Table 2). Among demographic and general clinical parameters, only number of drugs was weakly associated with HAQ-DI score (rho $=0.18, p=0.037)$. Two multiple regression models were generated for predicting HAQ-DI and ULS-8 scores. Depression, anxiety and patient global VAS scores remained significant for predicting HAQ-DI after multiple regression with covariates ULS-8, depression, anxiety, social support, patient and doctor global VAS scores, and number of drugs used (adjusted $\left.R^{2}=0.53, p<0.001\right)$. Although significant $(p<0.001)$, only $14 \%$ of variance in ULS-8 may be jointly explainable with covariates HAQ-DI, depression, anxiety, social support, doctor global VAS scores, number of drugs used, and education status. The only independent predictor was the education status. ULS-8 score did not correlate with DAS28, CDAI, and SDAI in RA; BASDAI, BASFI, and ASDAS in AS; and number of swollen and tender joints, ESR, CRP, patient global, doctor global, pain, and fatigue VAS scores in PSA.
Abstract THU0129 -Table 1.

Table 1. Demographic data, general clinical parameters, functional status, and ULS-8 scores according to disease groups.

\begin{tabular}{|c|c|c|c|c|}
\hline & $\begin{array}{c}\text { RA } \\
(n=58)\end{array}$ & $\begin{array}{c}\text { AS } \\
(n=53)\end{array}$ & $\begin{array}{c}\text { PsA } \\
(n=30)\end{array}$ & p value \\
\hline Age, years & $\begin{array}{c}58(\mathrm{QQR} \\
12)^{\mathrm{a}, \mathrm{b}}\end{array}$ & $\begin{array}{c}42(\text { IQR } \\
14)^{\mathrm{a}}\end{array}$ & $\begin{array}{c}45(\mathrm{IQR} \\
20)^{\mathrm{b}}\end{array}$ & $<0.001$ \\
\hline Female, $\mathbf{n}(\%)$ & $41(70,7)^{\mathrm{a}}$ & $19(35,8)^{a, b}$ & $21(70)^{b}$ & $<0.001$ \\
\hline Married, $\mathbf{n}(\%)$ & $46(79,3)$ & $46(86,8)$ & $28(93,3)$ & 0.196 \\
\hline Number of children & 2 (IQR 0$)$ & 2 (IQR 1) & 2 (IQR 1) & 0.102 \\
\hline Household size & 3 (IQR 2) & 4 (IQR I) & 4 (IQR 1) & 0.057 \\
\hline Education status, $\mathbf{n}(\%)$ & & & & $0.001^{\dagger}$ \\
\hline $\begin{array}{l}\text { Primary school or } \\
\text { lower }\end{array}$ & $33(56,9)^{a}$ & $13(24,5)^{a}$ & $14(46,7)$ & \\
\hline $\begin{array}{l}\text { Secondary or high } \\
\text { school }\end{array}$ & $17(29,3)^{a}$ & $28(52,8)^{\mathrm{a}}$ & $16(53,3)$ & \\
\hline Higher education & $8(13,8)$ & $12(22,6)^{\mathrm{a}}$ & $-a^{a}$ & \\
\hline Active & $16(27,6)^{2}$ & $31(58,5)^{2}$ & $10(33,3)$ & $.003^{\dagger}$ \\
\hline $\begin{array}{l}\text { Comorbid disease } \\
\text { present*, } n(\%)\end{array}$ & $36(62,1)^{a}$ & $19(35,8)^{a}$ & $12(40)$ & $0.014^{\dagger}$ \\
\hline$r$ of drugs & $5(\mathrm{IQR} 3)^{\mathrm{a}}$ & $2(\text { IQR } 1)^{a, b}$ & $5\left(\right.$ IQR 3) ${ }^{b}$ & $<0.001^{\dagger}$ \\
\hline gears & 8 (IQR 8) & 9 (IQR 9) & 9,5 (IQR 14) & 0.555 \\
\hline $\mathrm{ESR}, \mathrm{mm} / \mathrm{h}$ & $\begin{array}{c}25(I Q R \\
26)^{\mathrm{a}}\end{array}$ & $\begin{array}{c}13(I Q R \\
15)^{\mathrm{a}}\end{array}$ & 19 (IQR 22) & $0.014^{\dagger}$ \\
\hline CRP, $\mathbf{~ m g / L ~}$ & $6($ IQR 8,8) & $\begin{array}{c}7,6 \text { (IQR } \\
9,3)\end{array}$ & 5,1 (IQR 6) & 0.396 \\
\hline bioDMA & $6(10,3)^{a, b}$ & $37(69,8)^{2}$ & $16(53,3)^{b}$ & $<0.001$ \\
\hline $\begin{array}{l}\text { Adherent to treatment, } \\
\mathrm{n}(\%)\end{array}$ & $52(89,7)$ & $47(88,7)$ & $27(90)$ & 0.978 \\
\hline HAQ-DI groups, n(\%) & & & & 0.757 \\
\hline Mild & $52(89,7)$ & $48(90,6)$ & $29(96,7)$ & \\
\hline bility & $5(8,6)$ & $5(9,4)$ & $1(3,3)$ & \\
\hline Sev & $1(1,7)$ & - & - & \\
\hline ULS-8 score s $^{t}$ & 15 (IQR 7) & 15 (IQR 5) & 14,5 (IQR 7) & 0.749 \\
\hline $\begin{array}{l}\text { Continuous variables ar } \\
\text { (IQRs). Disease group, ag } \\
\text { comorbid disese, number } \\
\text { as appropriate. and }{ }^{b} \text { de } \\
\text { (post hoc adjusted } p<0.0 \\
\text { after control for confour } \\
\text { scores suggesting higher }\end{array}$ & $n=m$ & with & $\begin{array}{l}\text { quartile } \\
\text { tus, prese }\end{array}$ & \\
\hline
\end{tabular}

Abstract THU0129 -Table 2.

Table 2. Correlations between ULS-8, HAQ-DI, depression, anxiety, social support, and patient and doctor global VAS scores

\begin{tabular}{|c|c|c|c|c|c|c|c|}
\hline & & $\begin{array}{c}\text { Beck } \\
\text { depressi } \\
\text { on score }\end{array}$ & $\begin{array}{c}\text { Beck } \\
\text { anxiet } \\
y \\
\text { score } \\
\end{array}$ & $\begin{array}{c}\text { Social } \\
\text { support } \\
\text { score }\end{array}$ & $\begin{array}{l}\text { Patient } \\
\text { global } \\
\text { VAS } \\
\text { score }\end{array}$ & $\begin{array}{c}\text { Doctor } \\
\text { global } \\
\text { VAS } \\
\text { score } \\
\end{array}$ & $\begin{array}{l}\text { HAQ-DI } \\
\text { score }\end{array}$ \\
\hline $\begin{array}{l}\text { ULS-8 } \\
\text { score }\end{array}$ & $\begin{array}{r}\text { rho } \\
\text { p } \\
\text { value }\end{array}$ & $\begin{array}{c}0.32 \\
<0.001\end{array}$ & $\begin{array}{c}0.33 \\
<0.001\end{array}$ & $\begin{array}{l}-0.22 \\
0.009\end{array}$ & $\begin{array}{c}0.1 \\
0.224\end{array}$ & $\begin{array}{c}0.16 \\
0.058\end{array}$ & $\begin{array}{c}0.27 \\
0.001\end{array}$ \\
\hline $\begin{array}{l}\text { HAQ- } \\
\text { DI } \\
\text { score }\end{array}$ & $\begin{array}{r}\text { rho } \\
\mathbf{p} \\
\text { value }\end{array}$ & $\begin{array}{c}0.53 \\
<0.001\end{array}$ & $\begin{array}{c}0.53 \\
<0.001\end{array}$ & $\begin{array}{l}-0.25 \\
0.003\end{array}$ & $\begin{array}{c}0.5 \\
<0.001\end{array}$ & $\begin{array}{c}0.28 \\
<0.001\end{array}$ & - \\
\hline
\end{tabular}

Significant correlations exist between Beck depression, Beck anxiety,

social support, and patient and doctor global VAS scores.

Conclusion: Loneliness is associated with depression, anxiety, lack of social support, and lower education but not with disease activity in patients with inflammatory arthritis. Self -report loneliness, can be a contributing factor to the disability of the inflammatory arthritis.

\section{REFERENCE:}

[1] Kool MB, et al. Loneliness in patients with rheumatic diseases: the significance of invalidation and lack of social support. J Psychol 2012;146:229 41

Acknowledgement: no

Disclosure of Interests: Hakan Emmungil Grant/research support from: Roche, novartis, msd, Consultant for: roche, novartis, Speakers bureau: roche, novartis, msd, pfizer, celltrion, abbvie, Ufuk İlgen: None declared, Sezin Turan: None declared, Özge Kılıç: None declared

DOI: 10.1136/annrheumdis-2019-eular.7548

\section{THU0130 INCIDENCE AND RISK FACTORS OF FALLING IN PATIENTS WITH RHEUMATOID ARTHRITIS}

Rym Fakhfakh, Jguirim Mahbouba, Hibatallah Mosbeh, Abir Dghaies, Grassa Rim, Olfa Jmaa, Saoussen Zrour, Ismail Bejia, Mongi Touzi, Naceur Bergaoui. CHU Fattouma Bourguiba, Rheumatology, monastir, Tunisia

Background: People with rheumatoid arthritis (RA) may be at greater risk of falling than the non-RA population [1]. This increased falls risk may be due to RA disease-related impairments including pain, deformity and decreased muscle strength, as well as reduced functioning such as altered gait and a decline in postural stability. 